\title{
Sharpener blade, an unusual trachea-bronchial sharp foreign body in a child: a case report
}

\begin{abstract}
Foreign body (FB) aspiration is an extremely serious problem in childhood with varied clinical presentation demanding high degree of suspicion on the part of clinician. Here we discuss a case of 3year old child who aspirated a sharpener blade accidently while playing and was managed by bronchoscopy. Sharp and pointed foreign bodies in bronchus can result in potentially fatal complications. That can be removed safely by rigid bronchoscopy avoiding open surgical methods. There is only one and similar case reported in the literature previously as migratory foreign body bronchus with our best knowledge.
\end{abstract}

Keywords: foreign body, sharpener blade, bronchus, rigid bronchoscopy
Volume 6 Issue 3 - 2017

\author{
Bhushan Kathuria,' Dinesh Madhur, ${ }^{2}$ Himani \\ Dhingra, ${ }^{3}$ Mohit Pareek ${ }^{4}$ \\ 'Department of Otolaryngology Head Neck Surgery Aadhar \\ Hosital, India \\ ${ }^{2}$ Department of Otolaryngology Head Neck Surgery Agroha \\ medical college, India \\ ${ }^{3}$ Department of paediatric Sion hospital Mumbai, India \\ ${ }^{4}$ Department of Otolaryngology Head Neck Surgery Post \\ Graduate Institute of Medical Sciences, India
}

Correspondence: Bhushan Kathuria Consultant Department of Otolaryngology Head Neck Surgery Aadhar Hosital Hissar, India, Email kathuribhushan56@gmail.com

Received: December 27, 2016 | Published: March 09, 2017

\section{Introduction}

Accidental inhalation of both organic and non-organic foreign bodies continues to be a cause of childhood morbidity and mortality. ${ }^{1}$ The most common foreign bodies in children are coins, but marbles, button, batteries, safety pin and bottle tops have also been reported. While in adults, the most common foreign bodies are bones, dentures and metallic wires. ${ }^{2}$ Foreign bodies perforating the trachea ordinarily results in surgical emphysema or respiratory distress and tracheoesophageal fistula have been reported in literature.

\section{Case report}

A 3-year-old male child was referred to our institute with a history suggestive of sharpener blade aspiration accidently before 3 hours while playing. There was no history of vomiting, respiratory distress or any bleeding from mouth. On examination, vitals were stable and the child was breathing normally with no respiratory distress or cyanosis. On chest auscultation air entry on the left side was lessen as compared to the right side with added sounds (stridor or wheeze). On basis of history and physical examination foreign body aspiration was suspected and a chest $\mathrm{x}$-ray posterior-anterior view was done, which illustrate a radio-opaque rectangular foreign body in the region of the left main bronchus (Figure 1).

Rigid bronchoscopy was performed which revealed a piece of a sharpener blade in the left main bronchus which was impacted within the lumen and not completely blocking the distal airways. FB was grasped with a forceps and removed along with bronchoscope. It was rusted sharpener blade piece measuring size of $1 . \mathrm{cm} \times 0.6 \mathrm{~cm} \times 0.1$ $\mathrm{cm}$ (Figure 2). After removal of the foreign body, the bronchoscope was reintroduced looking for any airway mucosal injury which concealed no abnormality. The post-operative period was uneventful. A chest X-ray was taken at 6-8 hours post bronchoscopy to exclude the presence of pneumothorax or mediastinal emphysema and patient was discharged next day.

\section{Discussion}

The diagnosis and treatment of a child with a foreign body in the respiratory tract is a complex process, which necessitate close cooperation of a pediatric, a pulmonologist and a surgical team. The natural urge to explore the objects by mouth, lack of molar teeth, crying and playing while eating and lack of parental supervision contributes to this hazard in this age group. ${ }^{4}$ Adults may swallow nonfood objects accidentally or deliberately, when an underlying mental illness or a suicidal cause should be ruled out. An adequate positive history is very informative and helpful for diagnosis, but a negative history is present in about $15 \%$ of cases of foreign body aspiration. In the absence of positive history, a good clinical suspicion, physical examination, and chest $\mathrm{x}$-ray could make it possible to diagnose the case. $^{1}$

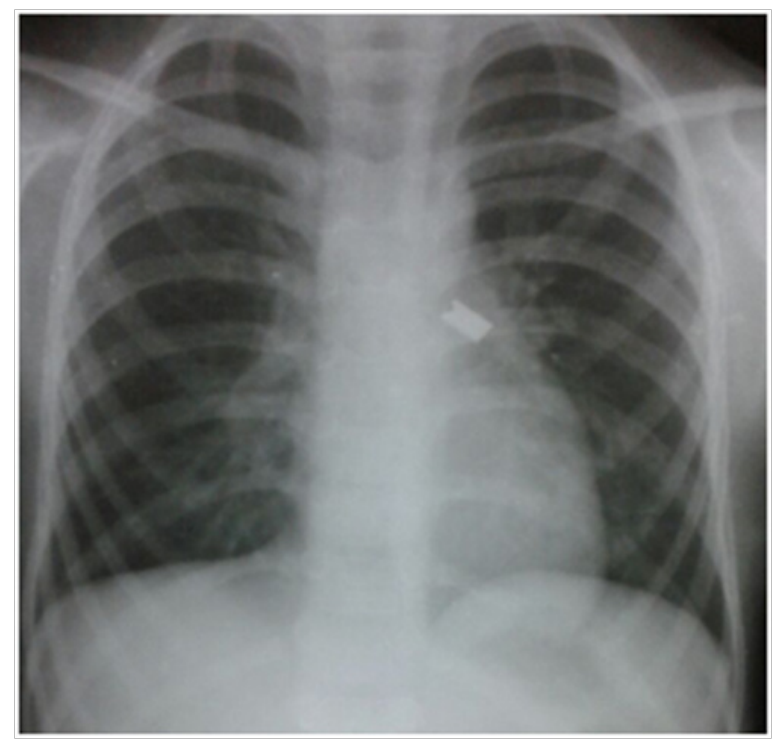

Figure I X-ray chet shows a radio-opaque forigen body (piece of sharpner blade) inhaled accidentally by 3 year old child.

A foreign body aspirated into the air passage can lodge in the larynx, trachea, or bronchi. Of these cases, $80 \%$ to $90 \%$ are found in the bronchi because their size and configuration allow passage through the larynx and trachea. Larger objects happen to impacted 
in the larynx or trachea, at times causing complete obstruction which is an acute emergency. In adults, bronchial foreign bodies tend to get lodged in the right main bronchus because of its lesser angle of convergence compared with the left bronchus and because of the location of the carina left of the midline. Because the angles made by the main stem bronchi with the trachea are identical until age $15 y e a r s$, foreign bodies are found on either side with equal frequency in patients in this age group $(<15$ years $) .^{5}$

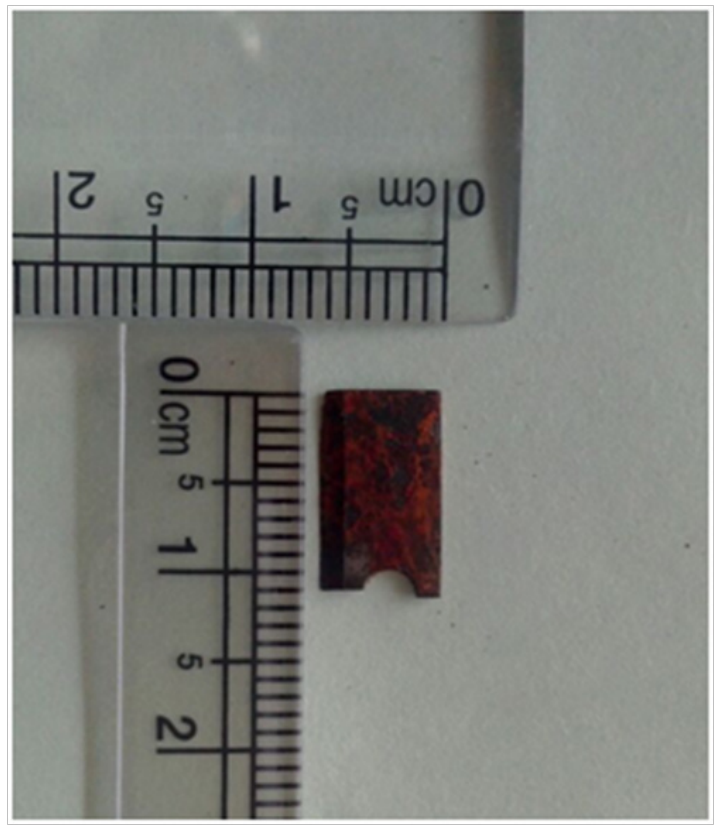

Figure 2 Image showing forigen body (rusted sharpener blade piece) measuring size approx. $1.1 \mathrm{~cm} \times 0.6 \mathrm{~cm} \times 0.1 \mathrm{~cm}$.

The main symptoms associated with aspiration are suffocation, cough, stupor, excessive sputum production, cyanosis or difficulty in breathing. These symptoms develop immediately after the aspiration. ${ }^{6}$ Management of inhaled foreign body depends on the type of foreign body and the site of impaction. Laryngeal and subglottic foreign bodies need urgent intervention in the form of tracheostomy or urgent bronchoscopy, whereas foreign bodies in the right or left main bronchus cause comparatively less airway problem. ${ }^{7}$

The organic foreign bodies have a tendency to readily immobilize. First, organic foreign bodies result in more severe mucosal inflammation leading to congestion and edema of the trachea-bronchial mucosa, and this may cause the development of granulation tissue around it. Second, some organic foreign bodies can absorb water and swell, resulting in total occlusion of the airway. On the other hand, inorganic foreign bodies are generally inert, and these may migrate even when they have been aspirated a long time previously. ${ }^{8}$

As most inhaled foreign bodies are radiolucent, clues as to their location on radiographs depends on secondary signs of complete or partial airway obstruction. The plain chest X-ray reveal radio-opaque foreign bodies in $23.56 \%$ of all patients with foreign bodies inhalation and many chest radiographs may have completely negative findings, especially within the first 24 hours following aspiration mainly in organic foreign body. ${ }^{6}$

The ideal means of foreign bodies removal is rigid bronchoscopy under general anesthesia with the removal rate of in $97.2 \%$ of patients with trachea-bronchial foreign bodies. ${ }^{8}$
The technique of anesthesia for foreign body removal in children is influenced by the general condition of the patient, the preference and experience of the anesthesiologist and the surgeon, and the type and location of the foreign body. ${ }^{9}$

For multiple pointed objects or objects with sharp cutting edges, a large diameter rigid bronchoscope can be used to protect the mucosa. No such protection is possible with the flexible endoscope. ${ }^{9}$ There is a significant risk of bronchial and tracheal laceration and perforation during extraction of such a foreign body with a flexible endoscope. It is a good tool for diagnosing and locating the sharp foreign body prior to extraction or for closely inspecting the tracheal and bronchial lumen, if necessary, following rigid endoscopic extraction.

After the bronchoscopy, the child should be observed in an anesthesia recovery room for stridor, respiratory distress or other signs suggestive of subglottic edema, damaged teeth, hemorrhage, bronchospasm and airway perforation. A chest radiograph should be obtained following bronchoscopy to exclude the presence of pneumothorax or mediastinal emphysema.

\section{Conclusion}

Foreign body in pediatric airway is a potentially life threatening situation, which requires emergency endoscopic removal. Foreign bodies in the airway continue to be a diagnostic and therapeutic challenge for the practicing otolaryngologist. Because a foreign body can mimic other conditions, particularly without a witnessed event, there can be a delay in management that may leads to complications. Sharp and pointed foreign bodies in bronchus can result in potentially fatal complications Also, sharpen foreign body may migrate from one main bronchus to other and produce respiratory obstruction of the previously normally ventilating lung. So, it should be considered as early as possible, requiring prompt recognition and early treatment to minimize the potentially serious and sometimes fatal consequences. However the most effective treatment of foreign body accidents is their prevention.

\section{Acknowledgments}

None.

\section{Conflicts of interest}

Author declares there are no conflicts of interest.

\section{Funding}

None.

\section{References}

1. Zerella JT, Dimler M, McGill LC, et al. Foreign body aspiration in children: value of radiography and complications of bronchoscopy. $J$ Pediatr Surg. 1998;33(11):1651-1654.

2. Mani N, Soma M, Massey S, et al. Removal of inhaled foreign bodiesmiddle of the night or the next morning? Int J Pediatr Otorhiolaryngol. 2009;73(8):1085-1089.

3. Henry WJ, Miscall L. Aortic-esophageal fistula. J Thorac Cardiovasc Surg. 1960;39(4):258-262.

4. Saki N, Nikalhlagh S, Rahim F, et al. Foreign body aspirations in infancy: a 20-year experience. Int J Med Sci. 2009;6(6):322-328.

5. Kim IG, Bremmitt WM, Humphrey A. Foreign body in the airway: a review of 202 cases. Laryngoscope. 1973;83(3):347-354. 
6. Kiyan G, Gocmen B, Tugtepe H, et al. Foreign body aspiration in children: The value of diagnostic criteria. Int J Pediatr Otorhinolaryngol. 2009;73(7):963-967.

7. Dunn GR, Wardrop P, Lo S, et al. Management of suspected foreign body aspiration in children. Clin Otolaryngol Allied Sci. 2004;27(5):384-386.
8. Rafanan AL, Mehta AC. Adult airway foreign body removal: what's new? Clinical Chest Med. 2001;22(2):319-330.

9. Divisi D, Di Tommaso S, Garramone M, et al. Foreign bodies aspirated in children: role of bronchoscopy. Thorac Cardiovasc Surg. 55(4):249-252. 